\title{
Sensitivity, Reproducibility, and Accuracy in Short Tandem Repeat Genotyping Using Capillary Array Electrophoresis
}

\section{Elaine S. Mansfield, ${ }^{1,3}$ Marina Vainer, ${ }^{1}$ Shellee Enad, ${ }^{1}$ David L. Barker, ${ }^{1}$ Dennis Harris, ${ }^{1}$ Eric Rappaport, ${ }^{2}$ and Paolo Fortina ${ }^{2}$}

\author{
${ }^{1}$ Molecular Dynamics, Inc., Sunnyvale, California 94086; ${ }^{2}$ The Children's Hospital of Philadelphia and \\ the University of Philadelphia, Abramson Pediatric Research Center, Philadelphia, Pennsylvania 19104
}

The Human Genome Initiative has increased significantly the rate at which disease-causing genes are being mapped and sequenced. New cost-effective methods to locate the genes and to characterize disease-causing mutations require robust, reproducible, and accurate protocols for measuring DNA fragment lengths. Capillary array electrophoresis (CAE) offers rapid, high-resolution separations, high throughput, and sensitive detection. To assess the utility of CAE for the accumulation of genetic information, we tested both sizing accuracy and reproducibility using 48-capillary prototype systems. Two multiplex PCR allelic ladder standards and several CA-repeat markers were analyzed in $>100$ runs. Reproducibility in typing $>8000$ genotypes reveals a standard deviation of less than $0.2 \mathrm{bp}$ on these systems under optimized conditions. However, sequence-dependent migration anomalies were observed at most simple sequence loci even when analyzed under denaturing conditions, resulting in a systematic bias in estimated fragment sizes. We show here that, by normalizing results to known typing controls, one can obtain locus-averaged accuracies of $<0.06 \mathrm{bp}$ and normalized results within $1 \mathrm{bp}$ of actual. We detect as little as a 1:30,000 dilution of a DNA quantitation standard stained with highly sensitive intercalating dyes, indicating an 80-zeptomole sensitivity limit. However, to obtain reproducible electrokinetic injection, $\sim 200$ attomoles of fluorescein-labeled DNA is required. These sensitivity limits, sizing precision, and accuracy, together with the 1-hr run times for 48-96 samples, indicate that CAE is a viable method for high-throughput genetic analysis of simple sequence repeat polymorphisms.

Short, tandemly repeated (STR) and highly polymorphic DNA markers have found extensive application rapidly in at least three important areas of applied biomedical research: the use of CArepeat polymorphisms to map disease causing genes (Weber and May 1989; Hearne et al. 1992); detection of expanded triplet repeat loci in diagnosis of several neurodegenerative diseases (Caskey et al. 1992); and the application of tetranucleotide STRs to human identification in forensic analysis (Edwards et al. 1991; Urquhart et al. 1995) or parentage testing (Edwards et al. 1991, 1992). Accurate typing of these markers requires high-resolution electrophoresis and comparison of fragment migration of marker DNA with reference standards to obtain reproducible size estimates.
${ }^{3}$ Corresponding author.

E-MAIL emansfield@mdyn.com; FAX (408) 773-8343.
Mapping unknown disease-causing genes involves identifying coinheritance of easy-to-type genetic markers with expression of the disease. Linkage analysis computer programs are used to test for evidence of colocalization by analyzing for concordance of specific CA-repeat alleles with disease in different families. When a marker is not very close to the disease gene, coinheritance of the marker and disease can be interrupted by meiotic recombination. High-resolution genetic maps make use of all available genetic typing markers including CA repeats, other STRs, sequence tagged sites, or restriction enzyme sites to tie the genetic map to physically mapped segments of DNA cloned from targeted regions. These cloned DNAs are then used to sequence the DNA and identify the underlying disease-causing gene. All of these steps require high-resolution separation techniques. Even moderate-scale gene mapping efforts involve the determination of tens of thousands of genotypes. Large-scale map- 
ping efforts or triplet repeat mutation screening efforts would require several million genotype determinations.

Expansion of unstable regions of triplet repeats in genomic DNA have been shown to underlie fragile $\mathrm{X}$ syndrome, Huntington disease, myotonic dystrophy, and at least eight other neurological disorders (Caskey et al. 1992). The expanded alleles at these disease loci show both somatic and germ-line instability, thus, allelic profiles can change over time (Eichler et al. 1994, 1995). In general, the length of expanded alleles correlates with disease status, although frequent monitoring is required because of the susceptibility to somatic mutation. Like analysis of CArepeat polymorphisms, diagnosis or monitoring progression of triplet repeat expansion disorders requires the reproducible sizing of large numbers of samples.

Analysis of STR polymorphisms is now applied to forensic human identification (Fregeau and Fourney 1993; Wiegand et al. 1993), and detection of fluorescently labeled PCR products has been accepted in this field due to the speed, sensitivity, throughput, and high discrimination that STR profiling provides (Kimpton et al. 1994). Several multicolor, multiplex STR reaction sets have been described for use on semi-automated, slab-gel systems (Fregeau and Fourney 1993; Urquhart et al. 1995; Sprecher et al. 1996). Again, in order to meet the volume of samples that must be screened in population-based studies for human identification, paternity testing, or linkage analysis, rapid electrophoresis of large numbers of samples is required. Bashkin et al. (1996b) described a capillary array instrument system that uses the format of 96-well microplates for sample loading to reduce labor costs and sample processing time and to improve sample tracking. We used that system for this study.

Capillary electrophoresis (CE) offers exquisite selectivity and very high-level resolution of closely related molecules. For example, oligonucleotides of identical composition but different primary sequence can be separated from one another (Konrad and Pentoney 1993). Similar results have been obtained separating peptides of identical composition but different primary sequence (Dolnik et al. 1993). We anticipated that the use of denaturing conditions during electrophoresis would minimize any composition or primary sequence effects on fragment mobility when analyzing simple sequence repeat DNA. However, many of the size estimates determined by capillary array electrophoresis (CAE) were biased systematically and reproducibly. We found that the sizing biases could be eliminated almost completely by normalizing results to genetic standards. By normalizing size estimates to standards of known size, results generated on different instruments or from multiple laboratories can be compared more easily. Here, we report analysis of $>8000$ genotypes at 12 STR loci, showing that CAE provides sizing precision of $<0.2 \mathrm{bp}$, a sensitivity of $<200$ attomoles of fluoresceinlabeled DNA or 80 zeptomoles of DNA stained with red intercalating dyes, and sizing accuracy within $1 \mathrm{bp}$ of actual when results are normalized to known typing controls.

\section{RESULTS}

\section{Sizing Reproducibility Studies}

The CTTV and FFFL fluorescent STR allelic ladders (Promega Corporation, Madison, WI) were processed multiple times to test reproducibility between capillaries within a run and between runs. An example set of six profiles of the CTTV ladder separated using a denaturing HEC matrix and CAE is illustrated in Figure 1. This ladder contains a mixture of 32 PCR products amplified from four different tetranucleotide loci ranging from 139 to 327 bp in size. In addition, we analyzed profiles of four polymorphic CA-repeat loci (D10S220, D13S143, D13S115, and D13S175). The repeat sequence, length, sizing accuracy (average deviation from actual in bp), and sizing precision (standard deviation in 36 replicates) are summarized in Table 1. Similar experiments were done using the FFFL allelic ladder standard containing 33 fragments ranging between 105 and $331 \mathrm{bp}$ amplified from four additional STR loci yielding similar sizing precision (data not shown).

Although the data consistently show excellent sizing precision (reproducibility), the sizing estimates were consistently high at each tetranucelotide repeat locus in the CTTV ladder. Confidence limits of $95 \%$ exclude the actual size from the determined range. For the CSF1PO marker, this deviation would be interpreted as the next higher molecular weight allele size. Precision for individual allelic determinations was highly reproducible. Size estimates for the D10S220 alleles determined using the ABI 373A (reference data) showed similar clustering above actual size, although the deviations from actual were more 


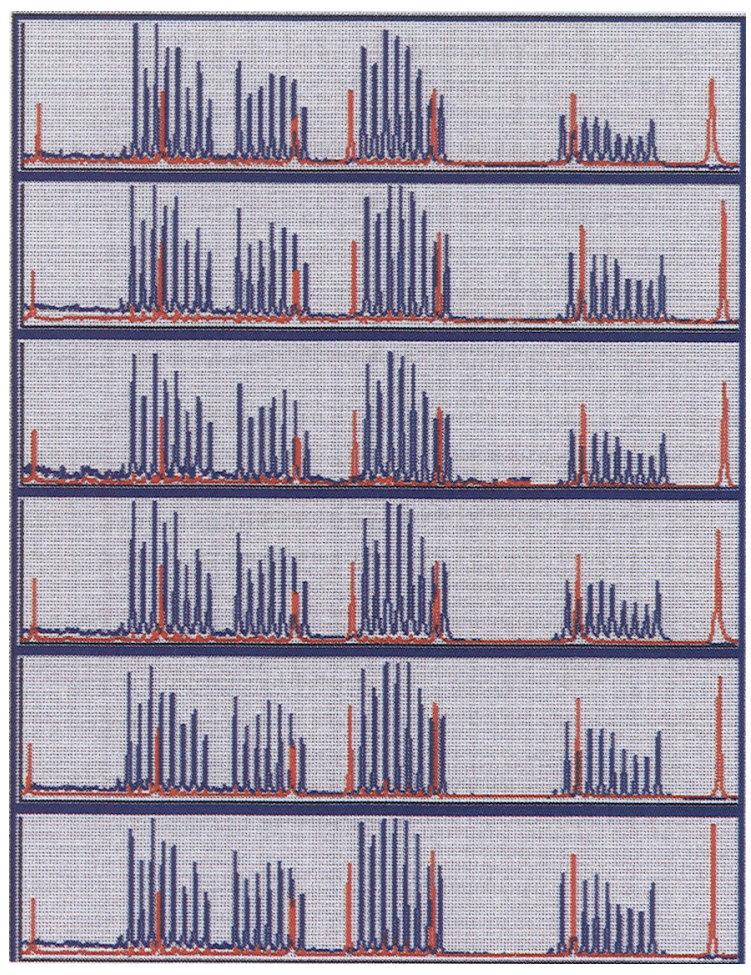

Figure 1 Capillary array electrophoresis profiles of fluorescein-labeled CTTV allelic ladder (Promega Corp., Madison, WI) comigrating with a tetramethyl rhodamine (TMR)-labeled sizing standard. Samples were prepared as described in the text and electrophoresed at $185 \mathrm{~V} / \mathrm{cm}$ for $1 \mathrm{hr}$. Emissions from the fluorescein-labeled allelic ladder were collected using a 520DF30 filter and a 535-nm beamsplitter in the optical path (blue trace in electropherograms). The TMR-labeled sizing standard emissions were collected using a 575DF30 filter in the second channel (red trace). Color separation and fragment sizing were carried out using ArrayQuant (Molecular Dynamics, Sunnyvale, CA). Fluorescence intensity profiles in six capillaries (1/16th of a 96-capillary run on a CAE system) are displayed as electropherogram plots of relative fluorescence intensity (vertical axis) vs. separation time (horizontal axis). Only fragments between 100 and $350 \mathrm{bp}$ are included in this display, and the seven fragments in the TMR50-350 standard (red trace) are 100, 150, 200, 220, 250, 300 , and $350 \mathrm{bp}$ in length. The CapillArray (Molecular Dynamics) program determines the sizes of the repeat alleles by comparing mobilities relative to the comigrating TMR50-350 standard.

modest (average difference was 0.28 vs. $0.56 \mathrm{bp}$ ). D10S220 profiles analyzed by CAE show excellent resolution of allelic products and PCR artifact bands observed with CA repeats (Weber and May 1989) (Fig. 2). Sizing precision in the ABI
373A reference data and CAE data were comparable (standard deviation of $0.16 \mathrm{vs} .0 .19 \mathrm{bp}$ ).

Capillary electrophoresis has been used to separate DNA on the basis of both size and composition (Wenz 1994). To test whether the observed deviation from actual size was due to the composition of the DNA sizing standard used, we compared computed size with actual size for the fragments in the CTTV ladder (Fig. 3). When size estimates were made using the Genescan350TMR size standard (Perkin Elmer, Foster City, CA) two problematic regions were clearly evident, in the vicinity of the 250-bp fragment and the 340bp fragment (Fig. 3A). A plot of size against retention time also shows significant deviation from linear for these two fragments (data not shown). For this reason, we specifically designed a new sizing standard for CAE, TMR50-350, which contains compositionally balanced fragments and yields excellent linearity of retention time with size $\left(R^{2}=0.9996-0.9999\right)$. When TMR50-350 was used to determine sizes of the CTTV ladder fragments, we observed excellent linear fit (Fig. 3B), although slight differences in slope and intercept of linear regression curves were obtained for each locus.

When comparing results using TMR50-350 with Genescan350-TMR, we obtain up to a tenfold better reproducibility of sizing estimates when using the former. This holds for several variations on the matrix formulation that we tested (data not shown). In addition, the accuracy of the estimates was also better (maximum size error was 5.60 bp for the TMR50-350 standard and 25.5 bp for the Genescan350 TMR standard). A major source of the computed sizing error using the $\mathrm{ABD}$ standard can be attributed to two fragments, at $250 \mathrm{bp}$ and $340 \mathrm{bp}$, that routinely migrate in a nonlinear fashion. Regression of computed size against actual for this standard consistently yields $\mathrm{R}^{2}>0.9956$. Removal of the 250-bp and 340-bp fragments from the standard curve improves the correlation $\left(\mathrm{R}^{2}=0.9989\right)$, although not to the level we obtain with the sizing standard developed for use in CAE.

\section{Sensitivity Studies}

Sensitivity estimates are influenced by the method of DNA labeling, the specific dyes used, and the detection optics of the instrument system (wavelength of excitation/emission; laser power; PMT settings; etc.). To determine sensitivity limits for end-labeled DNA analysis by CAE, 


\begin{tabular}{|c|c|c|c|c|c|}
\hline & D10S220 & vWA & TH01 & TPOX & CSF1PO \\
\hline Repeat & CA & AGAT & AATG & AATG & AGAT \\
\hline Size range (bp) & $102-120$ & $139-167$ & 179-203 & $224-252$ & $295-327$ \\
\hline \multicolumn{6}{|l|}{ Average deviation } \\
\hline from actual (bp) & +0.56 & +2.66 & +2.11 & +3.37 & +4.31 \\
\hline Standard deviation & 0.14 & 0.18 & 0.14 & 0.12 & 0.12 \\
\hline \multirow{9}{*}{ (bp) $(N=36)$} & 0.16 & 0.15 & 0.11 & 0.13 & 0.13 \\
\hline & 0.17 & 0.14 & 0.11 & 0.13 & 0.14 \\
\hline & 0.15 & 0.11 & 0.10 & 0.14 & 0.12 \\
\hline & 0.19 & 0.11 & 0.12 & 0.10 & 0.15 \\
\hline & 0.15 & 0.12 & 0.10 & 0.09 & 0.14 \\
\hline & 0.28 & 0.14 & 0.10 & 0.08 & 0.11 \\
\hline & 0.16 & 0.14 & 0.12 & 0.05 & 0.15 \\
\hline & 0.25 & 0.13 & 0.11 & 0.08 & 0.13 \\
\hline & 0.18 & 0.13 & 0.11 & 0.10 & 0.13 \\
\hline \multirow{2}{*}{$\begin{array}{l}\text { Average standard } \\
\text { deviation }\end{array}$} & & & & & \\
\hline & 0.187 & 0.131 & 0.110 & 0.102 & 0.134 \\
\hline
\end{tabular}

we ran a dilution series of a fluorescein-labeled sizing standard (Pharmacia Sizer 50-500, containing $5 \mathrm{fmole} / \mu \mathrm{l}$ of several fluorescein end-labeled fragments). In each run, this DNA yielded multiple peaks for each DNA segment under both denaturing and nondenaturing conditions (data not shown). Similar profiles were observed with other fluorescein-labeled DNA, notably a 20-bp ladder (MapMaker, BioVenture, Murfreesboro, $\mathrm{TN})$. A conservative estimate of detection limit of the fluorescein-labeled DNA would then be obtained because the fragments are split into multiple peaks. Our detection limit (signal/noise $>3$ ) for the Sizer 50-500 was 200 attomole per fragment when the standard was injected using our standard protocol. The same 200-attomole sample can, however, be injected five or more times. Consequently, we are actually detecting significantly less than 40 attomoles of fluorescein-labeled fragments on each injection.

A more sensitive method of detecting DNA on CAE is staining by intercalating dyes under nondenaturing conditions (McCord et al. 1993). Red-excitable dyes have been shown to have several advantages for use in DNA analysis by CAE. We found substantial improvement in signal-tonoise in both sequencing and fragment analysis applications by changing from a blue argon-ion laser to a lower-cost red HeNe laser. This is primarily because of substantially lower background fluorescence in the matrix, and glass capillary walls. When comparing performance of a series of proprietary red intercalating dyes with YoPro-3 (Molecular Probes, Eugene, OR), we observe a limit of detection (signal-to-noise ratio of 2.5) of the 200-bp fragment in DNA quantitation standard (Gibco BRL, Gaithersburg, MD) of $0.5 \mathrm{pg}$ (data not shown). This standard and a 100-bp ladder (Gensura, Del Mar, CA) can be detected in as little as a 1:30,000 dilution on CAE using $5 \mathrm{~nm}$ YoPro-3 or other red intercalating dyes excited with a $633 \mathrm{~nm}$ HeNe laser (R. Madabhushi, M. Vainer, V. Dolnik, S. Enad, D. Barker, D. Harris, and E. Mansfield, in prep.). This corresponds to a detection limit of 80 zeptomoles of DNA per band under these conditions. However, the disadvantage of using intercalating dyes for DNA staining is that nondenaturing separation conditions are required and multicolor analysis by CAE is impractical because the high field strengths applied during electrophoresis increase the off-rate of the dyes.

\section{Use of Reference DNA Standards to Normalize} Sizing Results

We observed very systematic sequence-dependent migration differences at most STR loci even when CAE separations were run under denaturing conditions. For example, when TBE was sub- 


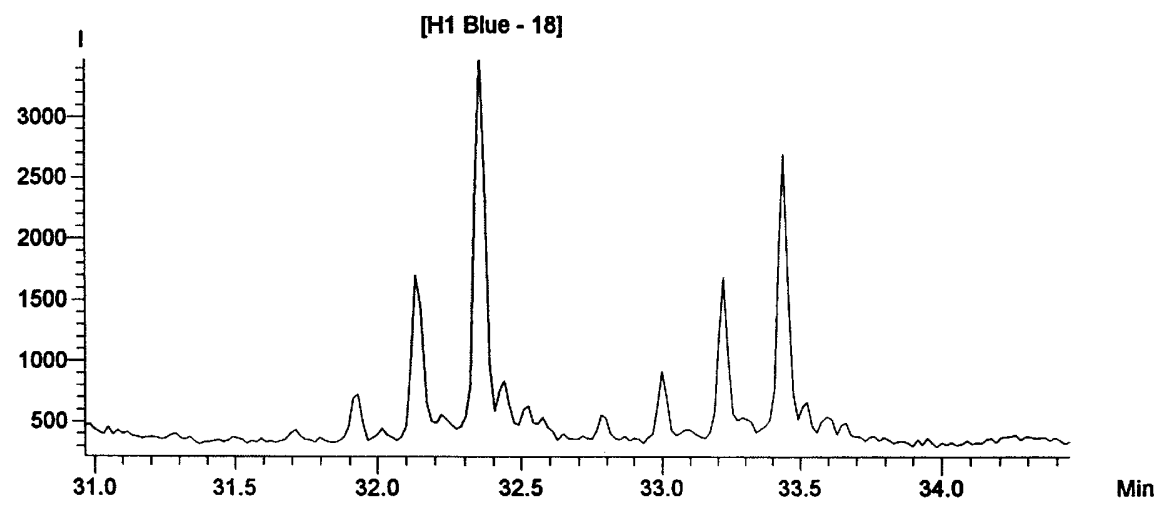

Figure 2 Resolution in a CA-repeat profile. PCR products amplified at the D10S220 locus (98-130 bp) were electrophoresed at $185 \mathrm{~V} / \mathrm{cm}$ using a scan rate of $1 \mathrm{scan} / \mathrm{sec}(1 \mathrm{~Hz})$. The HEX-labeled D10S220 DNA was coelectrophoresed with $0.2 \mu \mathrm{l}$ of a tetramethyl rhodamine-labeled (TMR) sizing standard. The dye-labeled DNA was excited using a 533-nm HeNe laser and emitted fluorescence separated using a $570-\mathrm{nm}$ beamsplitter in the optical path. HEXlabeled DNA emissions were collected using a 560DF15 filter, and TMR-labeled sizing standard emissions were collected using a 580DF30 in the second channel. Only data for the HEX-labeled channel are shown illustrating the profile of a heterozygous sample.

stituted with $100 \mathrm{~mm}$ TAPS [N-tris (hydroxymethyl) methyl-3-amino-propanesulfonic acid] in the matrix formulation, size estimates for CTTV alleles were 4.1-10.4 bp greater than actual (Table 2). However, we found that fragment measurements could be calibrated using known allele sizes to normalize results to the actual size. In this case, we determine an average ratio of the computed allele size relative to the actual size for each locus to obtain a normalizing factor. In the TrisTAPS buffer system, this ratio was 1.043 for vWA, 1.027 for TH01, 1.0368 for TPOX, and 1.032 for CSF1PO. To compute a normalized size, observed sizing results were divided by this normalizing factor. After normalization, we found that all measurements were within $0.68 \mathrm{bp}$ of actual (Table 2). The average normalized size of all fragments within 26 consecutive runs ranged from $-0.02 \mathrm{bp}$ to $+0.009 \mathrm{bp}$. The tight fit to actual indicates that the normalization process does not introduce any systematic sizing bias.

Rather than normalizing to an average of all alleles at a locus, it would be much more convenient to use genetic reference standard DNA genotypes such as Centre d'Etude du Polymorphisme Humain (CEPH) standards (Dib et al. 1996) to normalize CAE size estimates. We have found that both the running temperature and buffer composition of the matrix alter the degree of sizing bias observed in STR DNA separations
(M. Vainer, S. Enad, V. Dolnick, J. Bashkin, D. Harris, D.L. Barker, E.S. Mansfield, in prep.) and observed the minimum sizing biases at most loci using TBE buffer system. We amplified genetic typing control DNA at 12 separate loci, separated the PCR products using a TBE matrix and CAE, and then determined any bias in estimated sizes (Table 3 ). The calculated size estimates deviated $-3.30 \mathrm{bp}$ to $+4.35 \mathrm{bp}$ from actual among this test set of genetic loci. We calculated a normalization ratio for each locus using the known sizes of the control DNA and compared the accuracy of normalized and calculated sizes in up to 300 unknown DNA profiles at each locus. The accuracy of normalized sizes was consistently improved relative to the accuracy of the initial computed sizes (Table 3 ).

Excellent agreement of the averaged calibrated size to actual also indicates both accurate and precise measurements can be achieved by CAE. Within the normalized results, the maximum size difference remaining ranged from $+0.68 \mathrm{bp}$ to $-0.85 \mathrm{bp}(\mathrm{N}=8186$ genotypes). The average standard deviation of the normalized results ranged from $0.16 \mathrm{bp}$ for the vWA locus (size range is $139-167 \mathrm{bp}$ ) to $0.26 \mathrm{bp}$ for the CSF1PO locus (size range is $295-327 \mathrm{bp}$ ). Without normalizing results to the reference DNA, the greatest difference in size was observed at the CSF1PO locus (up to $+10.4 \mathrm{bp}$ ).

Linear normalization of simple sequence repeat loci shorter than $100 \mathrm{bp}$ in length did not work as well as for longer loci. To determine whether dye-induced migration differences may be contributing to this observation, we used the Applied Biosystems Matrix Standards (Perkin Elmer, Foster City, CA) to identify the extent to which the dyes change fragment mobility in CAE. The matrix standards contain sizing ladders labeled with the dye sets used in amidite, dye primer, and dye terminator chemistries. Differences in the position of individual peaks within CAE separated ladders would be attributable solely to the dyes at the end of each fragment 


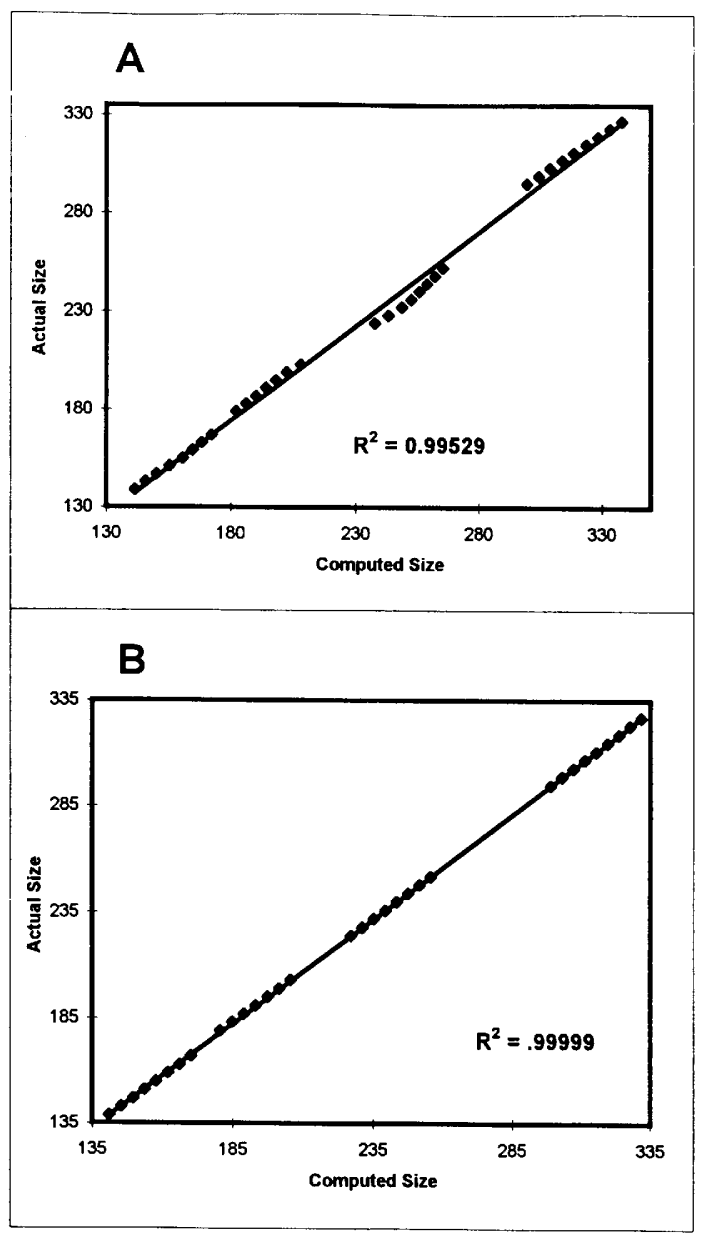

Figure 3 Comparison of estimated sizes of the 32 fragments in the CTTV allelic ladder (Promega Corp., Madison, WI) using different internal reference standards. The fluorescein-labeled CTTV ladder $(0.1 \mu \mathrm{l})$ was combined with $0.5 \mu \mathrm{l}$ of either Genescan350-TMR (Applied Biosystems Division, Perkin Elmer, Foster City, CA) $(A)$ or our own standard, TMR50-350 (B), and coelectrophoresed for 60 $\min$ at $185 \mathrm{~V} / \mathrm{cm}$. Fragment mobilities were converted into sizes using linear regression of retention time vs. size of fragments in the TMR-labeled sizing standard. The graph shows correlation of actual size vs. computed size for the CTTV ladder when each standard was used in the size calculations.

because the DNA in the ladders are identical within each set. We observed that fragments between 300 to $500 \mathrm{bp}$ comigrate in our CAE separations, although, for fragments outside this range, we observed discrepancies of $0.5-3 \mathrm{bp}$. The dye-induced migration differences were indeed nonlinear, in particular below 150 bp (Fig. 4), suggesting that the dyes could in part account for the poorer performance of linear normalization at shorter loci.

\section{DISCUSSION}

\section{Instrument Advantages}

Several groups have proposed using CE to increase the throughput of DNA fragment analysis. The difficulty with CE has been that only one capillary could be run and detected at a time, so that total throughput is no better than with slower slab gels with multiple lanes (Smith 1990). Despite this limitation, the extreme sensitivity, rapid run times, small sample size, and superior separation performance of capillary systems recently have been proven useful for identifying triplet repeat expansions in Kennedy disease (Nesi et al. 1994). Ultrathin slab gels $(50-100 \mu \mathrm{m})$ have some of the advantages of capillaries but are difficult to prepare and tedious to load with currently available instrumentation.

We have extended the work of R. Mathies and colleagues on the applying confocal fluorescence imaging of planar arrays of capillaries to DNA fragment analysis (Quesada et al. 1991; Rye et al. 1991; Huang et al. 1992; Clarke and Mathies 1994; Woolley and Mathies 1994). This technology offers an excellent opportunity to develop instrumentation capable of striking increases in DNA analysis efficiency and convenience. Molecular Dynamics has developed prototype instruments of this type capable of scanning across as many as 48 parallel capillaries (Bashkin et al. 1996b).

The labor-intensive steps of fragment separations are eliminated by CAE. Slab-gel preparation can be replaced by automated capillary filling using a low-viscosity gel matrix, refillable capillaries, and a pressurized gel filling station. The process is complete within 15 min without user intervention. Sample loading, another tedious step, is replaced with electrokinetic injection, thus, the increase in sample throughput is not limited to the electrophoresis time, but includes all steps of gel preparation and sample loading.

\section{Sensitivity}

McCord et al. (1993) and more recently Butler (1995) have reported using intercalating dyes to detect STR DNA using CE analysis. Intercalating dyes provide 10-50 times more sensitive detection than end-labeling in slab-gel electrophoresis 


\section{Table 2. Improved Accuracy of Sizing by Normalization of CTTV Ladder Fragments Separation in Matrix Containing Tris-TAPS Buffer System}

\begin{tabular}{|c|c|c|c|c|c|c|}
\hline Actual & $\begin{array}{l}\text { Calculated } \\
\text { size } \\
(N=22)\end{array}$ & $\begin{array}{l}\text { Standard } \\
\text { deviation }\end{array}$ & $\begin{array}{l}\text { Size } \\
\text { difference }\end{array}$ & $\begin{array}{l}\text { Calculated } \\
\text { size/actual }\end{array}$ & $\begin{array}{l}\text { Normalized } \\
\text { size }^{\mathrm{a}}\end{array}$ & $\begin{array}{l}\text { Normalized } \\
\text { size } \\
\text { difference }\end{array}$ \\
\hline \multicolumn{7}{|l|}{ vWA } \\
\hline 139 & 144.22 & 0.07 & 5.22 & 1.03 & 138.28 & -0.72 \\
\hline 143 & 148.77 & 0.10 & 5.77 & 1.04 & 142.64 & -0.36 \\
\hline 147 & 153.10 & 0.10 & 6.10 & 1.04 & 146.78 & -0.21 \\
\hline 151 & 157.38 & 0.10 & 6.38 & 1.04 & 150.89 & -0.10 \\
\hline 155 & 161.83 & 0.11 & 6.83 & 1.04 & 155.16 & 0.16 \\
\hline 159 & 166.15 & 0.12 & 7.15 & 1.04 & 159.30 & 0.29 \\
\hline 163 & 170.50 & 0.12 & 7.50 & 1.04 & 163.47 & 0.47 \\
\hline 167 & 174.90 & 0.13 & 7.90 & 1.04 & 167.68 & 0.68 \\
\hline \multicolumn{7}{|l|}{ TH01 } \\
\hline 179 & 183.08 & 0.10 & 4.08 & 1.02 & 178.28 & -0.71 \\
\hline 183 & 187.52 & 0.12 & 4.52 & 1.02 & 182.23 & -0.76 \\
\hline 187 & 191.97 & 0.14 & 4.97 & 1.02 & 186.95 & -0.05 \\
\hline 191 & 196.35 & 0.14 & 5.35 & 1.02 & 191.21 & 0.21 \\
\hline 195 & 200.78 & 0.13 & 5.78 & 1.02 & 195.55 & 0.55 \\
\hline 199 & 204.82 & 0.13 & 5.82 & 1.02 & 199.50 & 0.50 \\
\hline 203 & 208.96 & 0.14 & 5.96 & 1.02 & 203.59 & 0.59 \\
\hline \multicolumn{7}{|l|}{ TPOX } \\
\hline 224 & 232.26 & 0.15 & 8.26 & 1.03 & 224.02 & 0.02 \\
\hline 228 & 236.40 & 0.17 & 8.40 & 1.03 & 228.01 & 0.01 \\
\hline 232 & 240.60 & 0.21 & 8.60 & 1.03 & 232.06 & 0.06 \\
\hline 236 & 244.74 & 0.21 & 8.74 & 1.03 & 236.05 & 0.05 \\
\hline 240 & 248.93 & 0.21 & 8.93 & 1.03 & 240.09 & 0.09 \\
\hline 244 & 252.98 & 0.18 & 8.98 & 1.03 & 244.01 & 0.005 \\
\hline 248 & 257.06 & 0.23 & 9.06 & 1.03 & 247.93 & -0.06 \\
\hline 252 & 261.08 & 0.23 & 9.08 & 1.03 & 251.81 & -0.18 \\
\hline \multicolumn{7}{|l|}{ CSF1PO } \\
\hline 295 & 304.49 & 0.11 & 9.49 & 1.03 & 295.03 & 0.03 \\
\hline 299 & 308.70 & 0.16 & 9.70 & 1.03 & 299.11 & 0.11 \\
\hline 303 & 312.70 & 0.20 & 9.70 & 1.03 & 302.99 & -0.01 \\
\hline 307 & 316.82 & 0.13 & 9.82 & 1.03 & 306.98 & -0.02 \\
\hline 311 & 320.96 & 0.12 & 9.96 & 1.03 & 310.99 & -0.01 \\
\hline 315 & 325.07 & 0.10 & 10.07 & 1.03 & 314.98 & -0.02 \\
\hline 319 & 329.24 & 0.08 & 10.24 & 1.03 & 319.02 & 0.01 \\
\hline 323 & 333.33 & 0.10 & 10.33 & 1.03 & 322.97 & -0.02 \\
\hline 327 & 337.42 & 0.09 & 10.42 & 1.03 & 326.93 & -0.06 \\
\hline Average & & 0.14 & 7.78 & & & 0.01 \\
\hline Maximum & & 0.23 & 10.41 & & & 0.68 \\
\hline Minimum & & 0.07 & 4.08 & & & -0.76 \\
\hline
\end{tabular}

a Normalized size $=$ (calculated size)/(locus average normalization ratio) (average of the values in the fifth column for each locus). The normalization ratio was 1.043 for VWA, 1.027 for TH01, 1.0368 for TPOX, and 1.032 for CSF1PO. See text for more details.

applications (Mansfield et al. 1993). Similarly, M. Marino, L. Tully, J.M. Devaney, S. Del Rio, and J. Girard (pers. comm.) observe a tenfold greater sensitivity using TOTO-1 than end-labeled PCR products analyzed by CE. Another ideal intercalating dye is thiazole orange (Becton Dickinson,
San Jose, CA), because it does not cross-link DNA molecules in a mixture. Previous research at Molecular Dynamics demonstrated that the detection limit for thiazole orange-labeled-DNA on the CAE system is $\sim 500$ times greater than ethidium bromide staining and detection on a UV lightbox 


\section{Table 3. Computed and Normalized Sizing Results at 12 Short Tandem Repeat Loci Separation in Matrix Containing Tris-Borate EDTA Buffer System}

\begin{tabular}{lccc}
\hline Locus & Size range & $\begin{array}{c}\text { Average deviation } \\
\text { in size (bp) }\end{array}$ & $\begin{array}{c}\text { Normalized size } \\
\text { deviation (bp) }\end{array}$ \\
\hline VWA & $139-167$ & 2.66 & 0.012 \\
TH01 & $179-203$ & 2.27 & -0.057 \\
TPOX & $224-252$ & 3.37 & 0.0013 \\
CSF1PO & $295-327$ & 4.35 & 0.0001 \\
D10S220 & $98-130$ & 0.56 & 0.0007 \\
LPL & $105-129$ & -2.06 & 0.022 \\
F13B & $169-189$ & -0.54 & 0.0017 \\
FESFPS & $222-250$ & 0.23 & 0.016 \\
F13AD1 & $283-331$ & -1.05 & 0.053 \\
D13S143 & $131-137$ & -2.49 & 0.018 \\
D13S115 & $165-179$ & -3.30 & -0.004 \\
D13S175 & $255-267$ & -3.04 & 0.008 \\
\hline
\end{tabular}

(R. Johnston, unpubl.). For example, 1:500 dilutions of most commercially available DNA standards yield peaks between 300 and 1000 relative fluorescence units ( $\mathrm{rfu}$ ) on the CAE system using

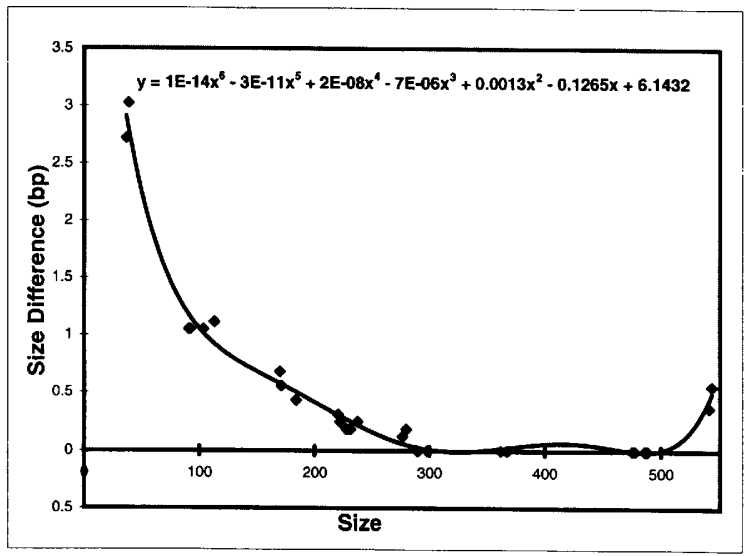

Figure 4 Sixth-order curve fit to dye-induced migration anomalies in CAE. A 26-fragment ladder labeled with 6-carboxy fluorescein (6-FAM) and tetramethyl rhodamine (TMR) were coelectrophoresed to determine sizing differences imparted by the dyes. The DNA was separated using the conditions descried in the text and signals detected as described in Fig. 1. Peak positions were determined following color separation in ArrayQuant. Sizes of the TMR-labeled fragments were used to compute effective size of the 6-FAM-labeled fragments. The difference in estimated size over the range of the ladder is graphed and fit to a sixth-order curve using Microsoft Excel. the staining conditions of Bashkin et al. (1996b) $(0.4 \mu \mathrm{M}$ thiazole orange in the matrix and running buffer; data not shown). Here we report even more substantial sensitivity gains by using red intercalating dyes and CAE. Lower dye concentrations were required (1-10 nM) and we detected a $1: 30,000$ dilution of a DNA quantiation standard using red excitable dyes, indicating an 80-zeptomole sensitivity limit under these conditions.

Sizing Accuracy and Precision

CAE offers extremely highresolution separations. We observed locus-specific sizing biases at each simple sequence repeat locus tested. Consequently, we designed experiments specifically to test the contribution of several factors to both precision (reproducibility) and accuracy (ability to obtain the correct fragment length). Those factors contributing to errors in fragment sizing estimates include:

- Composition or sequence-specific effects

- The composition or fragment spacing in the DNA sizing standard

- Mobility shifts due to the dyes used to label the DNA fragments

- Resolution range of the matrix

- The curve fitting algorithm used-i.e., linear interpolation or fit to other curves

- Sampling rate (scan speed) to accurately locate the center of peaks in both the standard and the unknowns

Unusually rapid migration of simple sequence DNA has been observed previously in separations both by slab-gel electrophoresis and CE. Chastain et al. (1995) observed a $20 \%$ difference in migration of triplet repeat DNA from the FMR1 and $S C A 1$ genes separated in nondenaturing polyacrylamide gels. The anomalous migration was found to depend on the number of triplet repeats and the length of flanking DNA, as well as on the composition and running temperature of the gels. Wong et al. (1995) similarly reported up to $60 \%$ migration bias when separating triplet re- 
peat DNA from the myotonic dystrophy locus, which they correct by normalizing to known size. The latter study was made using agarose-gel electrophoresis and measurements made on autoradiograms of Southern blots. The log transformation method used by this group to normalize the anomalous fragment migration is not suitable for separations made in real time (detection at a fixed migration distance as in CAE). However, this work does set a precedent for the use of size normalization to correct for migration differences observed in electrophoretic separations of simple sequence repeat fragments.

The systematic, anomalous rapid electrophoretic mobility of simple sequence DNA also has been observed resently in slab-gel separations of triplet repeat DNA (Chastain et al. 1995). In this study, a DNA fragment containing a CTG triplet repeat from fragile $X$ gene migrated up to $20 \%$ faster in nondenaturing polyacrylamide gels. The amount of anomalous migration was also dependent upon both the number of repeats and the length of the flanking DNA in the PCR products. We did not observe as dramatic a shift in migration of the repeat DNA using CAE under denaturing conditions ( $10 \%$ formamide, $6 \mathrm{M}$ urea), although the CAE results are consistent with the results obtained using nondenaturing polyacrylamide gels. For example, we observe up to 9-bp sizing bias ( $3 \%$ at $300 \mathrm{bp})$ as a consequence of faster migration of simple sequence DNA analyzed by CAE.

Fregeau and Fourney (1993) observed differences in computed sizes of simple sequence DNA using real-time fluorescence detection and the ABI 373A. The authors noted a 6.4-bp difference in computed size at the THO1 locus (size range $=179-203 \mathrm{bp}$ ) when nondenaturing gels were compared with denaturing conditions. The authors also found that longer STR loci had more pronounced differences. Although CSF1PO was not studied by this group, results at the ARA triplet repeat locus (which has a similar length to CSF1PO but contains a different repeat) show up to 14-bp difference in computed size under native gel conditions. The authors prefer to use native gel separations on that system because more rapid runs could be achieved (6-9 hr by conventional electrophoresis vs. $1 \mathrm{hr}$ by CAE).

The degree of anomalous migration in capillary electrophoresis is impacted significantly by the labeling method and sizing standard used during detection. For example, in a systematic study of parameters influencing CE sizing accu- racy and precision, Butler (1995) observed a 4.8bp difference at the TH01 locus when heterologous flanking DNA standards were used to estimate size. However, when measurements were made on TH01 allelic standards, unknown samples size within $0.2 \mathrm{bp}$ of the allelic standards, resulting in very accurate typing and allele calling. In their study, a single intercalating dye (YoPro-1, Molecular Probes) was used to label both the standard and the TH01 PCR products, indicating that dye-induced migration difference is not causing anomalous migration differences between the simple sequence DNA and the standard.

By normalizing CAE measurements to confirmed sizes of genetic typing standards within the run, we reduced the average, normalized size difference at CSF1PO from up to $10.4 \mathrm{bp}$ to 0.14 bp. Together, these results indicate that sizing measurements made using CAE are both consistent with and as good as those reported by expert laboratories in the field. In addition, the use of software and reference DNA standards to normalize sequence-specific measurement errors should enable a more direct comparison of genetic typing results between laboratories or for computation of test results in a forensic context (Kuffner et al. 1996).

Knowles et al. (1992) first proposed a method for interlaboratory standardization of linkage results using CEPH DNA typing standards. These DNAs would be included in each analysis to eliminate variability of both labeling and PCR artifacts. Allele sizes of a CEPH control DNA are now published at 5264 mapped, polymorpic, microsatellite loci (Dib et al. 1996). Our implementation of genetic typing standards to normalize size estimates eliminates a substantial proportion of sequence-specific migration artifacts observed with repetitive markers. When analyzing over 8000 STR genotypes by CAE, all normalized results were within $1 \mathrm{bp}$ of actual, and locus averaged results were within $0.05 \mathrm{bp}$. The within-run precision (reproducibility estimated by standard deviation) averaged $0.13 \mathrm{bp}$, and between-run precision was $0.18 \mathrm{bp}$. We achieve a detection limit of 40 attomoles of fluorescein end-labeled DNA and 80 zeptomoles of DNA stained with intercalating dyes. This high sensitivity translates to lower reagent consumption (for the CTTV allelic ladder standard we use $0.1 \mu \mathrm{l} /$ capillary load vs. $2.5 \mu \mathrm{l}$ load recommended for other fluorescent instrument systems). We conclude that these data suggest CAE is a viable method to 


\section{MANSFIELD ET AL.}

achieve rapid, sensitive, reliable, and reproducible genotyping results of STR and microsatellite polymorphisms.

\section{METHODS}

We used a 48-capillary array prototype system described previously by Bashkin et al. (1996b) to separate fluorescently labeled DNA fragments. The internal walls of polyimide-coated capillaries ( $200 \mu \mathrm{m}$ O.D, $75 \mu \mathrm{m}$ I.D; Polymicro Technologies, Phoenix, AZ) were coated with polyacrylamide to prevent electro-osmotic flow during electrophoresis using the procedure of Cobb et al. (1990). Coated capillaries were cut to $65 \mathrm{~cm}$ and assembled into arrays of 16 capillaries with detection windows burned at $40 \mathrm{~cm}$ path length. We modified the hydroxyethyl cellulose (HEC) DNA sequencing matrix of Bashkin et al. (1996a). Matrix was introduced into the capillary at 400 psi for $2 \mathrm{~min}$, allowed to equilibrate with ambient pressure for $5 \mathrm{~min}$, and pre-electrophoresed for $10 \mathrm{~min}$ at 185 volts $/ \mathrm{cm}$.

In our protocols, all samples were heat-denatured at $90^{\circ} \mathrm{C}$ for $5 \mathrm{~min}$ in $50 \%(\mathrm{vol} / \mathrm{vol})$ formamide prior to injection into the capillaries. DNA was introduced into the capillaries by electrokinetic injection for $15 \mathrm{sec}$ at $14 \mathrm{kV}$. In the reproducibility and accuracy studies, we used PCRgenerated allelic ladder standards provided in GenePrint STR System fluorescent typing kits (Promega Corporation, Madison, WI). The CTTV STR standard ladder contains fluorescein-labeled PCR products representing all common alleles at the CSF1PO (295-327 bp), TPOX (224-252 bp), TH01 (179-203 bp), and vWA (139-167 bp) polymorphic loci. The FFFL standard contains fluorescein-labeled allelic products amplified at the F13AD1 (283-331 bp), FESFPS (222-250 bp), F13B (169-189 bp), and LPL (105-129 bp) loci. A $0.1 \mu \mathrm{l}$ aliquot of the fluorescein-labeled ladder was combined with $0.5 \mu$ l tetramethyl rhodamine-labeled sizing standard (Genescan 350-TMR; Perkin Elmer) in a total volume of $3 \mu \mathrm{l}$ of $50 \%(\mathrm{vol} / \mathrm{vol})$ deionized formamide. For most experiments, we substituted a new tetramethyl rhodamine-labeled sizing standard that we specifically formulated for CAE separations. This standard contains eight compositionally balanced fragments between 50 and 350 bp in length and was formulated to minimize sequencespecific migration anomalies observed in CE (Wenz 1994). DNA fragments were separated for $60-70 \mathrm{~min}$ at $185 \mathrm{~V} / \mathrm{cm}$ field strength. Fresh matrix was introduced into the capillaries between runs as described by Bashkin et al. (1996a). Data were analyzed with prototype fragment analysis programs developed at Molecular Dynamics, ArrayQuant and CapillArray, and with Excel (Microsoft, Seattle, WA). Typing results determined on the CAE system were compared with manual ${ }^{32} \mathrm{P}$-PCR results and with those obtained by use of the Applied Biosystems Model 373A semiautomated fluorescent sequencer (ABI 373A).

Sensitivity studies made use of other fluorescently labeled DNA standards or DNA quantitation standards detected with intercalating dyes as cited in the text. We used protocols similar to M. Marino, L. Tully, J.M. Devaney, S. Del Rio, and J. Girard (pers. comm.) and a dilution series of DNA quantitation standards (Gibco BRL; GenSura) to determine the limit of detection of DNA fragments electrokinetically injected into the CAE prototype systems.
We modified the method of Bashkin et al. (1996b) and added $0.4 \mu \mathrm{M}$ thiazole orange (Becton Dickinson, San Jose, CA) to the matrix and running buffer of a nondenaturing matrix [0.5-2\% hydroxyethyl cellulose (Polysciences, Warrington, PA), $44 \mathrm{~mm}$ Tris-base, $44 \mathrm{~mm}$ boric acid, and 0.5 mM EDTA (pH 8.3)] when detecting unlabeled DNA quantitation standards on the CAE systems using a $20-\mathrm{mW}$ argon-ion laser. Molecular Probes, Inc., provided us with a series of proprietary red intercalating dyes to evaluate by $\mathrm{CAE}$. These longer wavelength dyes were excited by use of a separate prototype system with a $10-\mathrm{mW} 633-\mathrm{nm} \mathrm{HeNe}$ laser. The same matrix was used except that the dyes were diluted to a final concentration of 2-5 nм. We analyzed and detected a range of DNA sizing and quantitation standards diluted in the range of 1:500 to 1:30,000 in the sensitivity studies.

\section{ACKNOWLEDGMENTS}

This work was supported by Molecular Dynamics and a small business research innovation (SBIR) grant \#1R43NS/ MH34589-01 awarded by the National Institutes of Health to E.S.M. Additional support was provided by an advanced technology grant \#70NANB5H1031 awarded by the National Institute of Standards and Technology (NIST) to D.L.B. We thank Drs. John Bashkin and Richard Johnston for assistance with operating the CAE prototype units and Michele Marsh for technical assistance.

The publication costs of this article were defrayed in part by payment of page charges. This article must therefore be hereby marked "advertisement" in accordance with 18 USC section 1734 solely to indicate this fact.

\section{REFERENCES}

Bashkin, J., M. Marsh, D. Barker, and R. Johnston. 1996a. DNA sequencing by capillary electrophoresis with a hydroxyethylcellulose sieving buffer. Appl. Theoret. Electrophoresis 6: 23-28.

Bashkin, J., D. Roach, J. Leong, D. Barker, and R. Johnston. 1996b. Implementation of a capillary array electrophoresis instrument. J. Capil. Electrophoresis (in press).

Butler, J.M. 1995. Sizing and quantitation of polymerase chain reaction products by capillary electrophoresis for use in DNA typing. Ph.D. dissertation, University of Virginia, Charlottesville, VA.

Caskey, C.T., A. Pizzuti, Y.-H. Fu, R.G. Fenwick, and D.L. Nelson. 1992. Triplet repeat mutations in human disease. Science 256: 784-789.

Chastain, P.D., E.E. Eichler, S. Kang, D.L. Nelson, S.D. Levene, and R.R. Sinden. 1995. Anomalous rapid electrophoretic mobility of DNA containing triplet repeats associated with human disease genes.

Biochemistry 34: 16125-16131.

Clark, S.M. and R.A. Mathies. 1993. High-speed parallel separation of DNA restriction fragments using capillary array electrophoresis. Anal. Biochem. 215: 163-170.

Cobb, K.A., V. Dolnik, and M. Novotny. 1990.

Electrophoretic separation of proteins in capillaries with 


\section{CA-REPEAT AND STR GENOTYPING IN CAPILLARY ARRAYS}

hydrolytically stable surface structure. Anal. Chem. 62: $2478-2483$.

Dib, C., S. Faure, C. Fizames, D. Samson, A. Drout, A. Vignal, P. Millasseau, S. Marc, J. Hazan, E. Seboun, M. Lanthrop, G. Gyapay, J. Morissette, and J. Weissenbach. 1996. A comprehensive genetic map of the human genome based on 5,264 microsatellites. Nature 380: A4-A128.

Dolnik, V., M. Novotny, and J. Chmelin. 1993. Electromigration behavior of poly-(L-glutamate) conformers in concentrated polyacrylamide gels. Biopolymers 33: 1299-1306.

Edwards, A., A. Civitello, H.A. Hammond, and C.T. Caskey. 1991. DNA typing and genetic mapping with trimeric and tetrameric tandem repeats. Am. J. Hum. Genet. 49: 746-756.

Edwards, A., H.A. Hammond, L. Jin, C.T. Caskey, and R. Charkaborty. 1992. Genetic variation at five trimeric and tetrameric tandem repeat loci in four human population groups. Genomics 12: 241-253.

Eichler, E.E., J.J.A. Holden, B.W. Popovich, A.L. Reiss, K. Snow, S.N. Thibodeau, C.S. Richards, P.A. Ward, and D.L. Nelson. 1994. Length of uninterrupted CGG repeats determines instability in the FMR1 gene. Nature Genet. 8: 88-94.

Eichler, E.E., H.A. Hammond, J.N. Macpherson, P.A. Ward, and D.L. Nelson. 1995. Population survey of the human FMR1 CGG repeat substructure suggests biased polarity for the loss of AGG interruptions. Hum. Mol. Genet. 4: 2199-2208.

Fregeau, C.J. and R.M. Fourney. 1993. DNA typing with fluorescently tagged short tandem repeats: A sensitive and accurate approach to human identification. BioTechniques 15: 100-119.

Hearne, C.M., S. Ghosh, and J.A. Todd. 1992. Microsatellites for linkage analysis of genetic traits. Trends Genet. 8: 288-294.

Huang, X.C., M.A. Quesada, and R.A. Mathies. 1992. Capillary array electrophoresis using laser-excited confocal fluorescence detection. Anal. Chem. 64: $2149-2154$.

Kimpton, C.P., D. Disher, S. Watson, M. Adams, A. Urquhart, J. Lygo, and P. Gill. 1994. Evaluation of an automated DNA profiling system employing multiplex amplification of four tetrameric STR loci. Int. J. Legal Med. 106: 302-311.

Knowles, J.A., V.J. Vieland, and T.C. Gilliam. 1992. Perils of gene mapping with microsatellite markers. Am. J. Hum. Genet. 51: 905-908.

Konrad, K.D. and S.L. Pentoney. 1993. Contribution of secondary structure to DNA mobility in capillary gels. Electrophoresis 14: 502-508.
Kuffner, C.A., E. Marchi, J.M. Morgado, and C.R. Rubio. 1996. Capillary electrophoresis and Daubert: Time for admission. Anal. Chem. 68: 241A-246A.

McCord, B.R., D.L. McClure, and J.M. Jung. 1993. Capillary electrophoresis of polymerase chain reaction-amplified DNA using fluorescence detection with an intercalating dye. J. Chrom. 652: 75-82.

Mansfield, E.S., J. Robertson, R. Lebo, M. Lucero, P.E. Mayrand, E. Rappaport, M. Sartore, T. Parrella, S. Surrey, and P. Fortina. 1993. Duchenne/Becker muscular dystrophy carrier detection using quantitative PCR and fluorescence-based strategies. Am. J. Med. Genet. 48: $200-208$.

Nesi, M., P.G. Righetti, M.C. Patrosso, A. Ferlini, and M. Chiari. 1994. Capillary Electrophoresis of polymerase chain reaction-amplified products in polymer networks-The case of Kennedy disease. Electrophoresis 15: 644-646.

Quesada, R.A., H.S. Rye, J.C. Gingrich, A.N. Glazer, and R.A. Mathies. 1991. High-sensitivity detection with a laser-excited confocal fluorescence gel scanner. BioTechniques 10: 616-621.

Rye, H.S., R.A. Quesada, K. Peck, R.A. Mathies, and A.N. Glazer. 1991. High-sensitivity two-color detection of double-stranded DNA with a confocal fluorescence gel scanner using ethidium homodimer and thiazole orange. Nucleic Acids Res. 19: 327-338.

Smith, L.M. 1990. Automated DNA sequencing and the analysis of the human genome. Genome 31: 929-937.

Sprecher, C.J., C. Pures, A.M. Lins, and J.W. Schumm. 1996. General approach to analysis of polymorphic short tandem repeat loci. BioTechniques 20 266-276.

Urquhart, A., N.J. Oldroyd, C.P. Kimpton, and P. Gill. 1995. Highly discriminating heptaplex short tandem repeat PCR system for forensic identification. BioTechniques 18: 116-128.

Weber, J.L. and P.E. May. 1989. Abundant class of human DNA polymorphisms which can be typed using the polymerase chain reaction. Am. J. Hum. Genet. 44: $388-396$.

Wiegand, P., B. Budowle, S. Rand, and B. Brinkmann. 1993. Forensic validation of the STR systems SE33 and TC11. Int. J. Legal Med. 105: 315-320.

Wenz, M. 1994. Capillary electrophoresis as a technique to analyze sequence-induced anomalously migrating DNA fragments. Nucleic Acids Res. 22: 4002-4008.

Wong, L.-J.C., T. Ashiwawa, D.G. Monckton, C.T. Caskey, and C.S. Richards. 1995. Somatic heterogeneity of the CTG repeat in myotonic dystrophy is age and size dependent. Am. J. Hum. Genet. 56: 114-122.

Woolley, A.T. and R.A. Mathies. 1994. Ultra-high-speed DNA fragment separations using microfabricated capillary array electrophoresis chips. Proc. Natl. Acad. Sci. 91: 11348-11352.

Received June 27, 1996; accepted in revised form July 29, 1996. 